\section{TWO CASES OF VEIN-GRAFTING FOR THE MAINTENANCE OF A DIRECT ARTERIAL CIRCULATION.}

\author{
BY J. HOGARTH PRINGLE, M.B., C.M. EDIN., \\ F.R.C.S. ENG.,
}

SURGEON TO THE GLASGOW ROYAL INFIRMARY.

THE reports of the two following cases are published as examples of the successful application, in human surgery, of the lessons that have been taught by the splendid work of Carrel in his experimental studies on the surgery of the wascular system. They are not the first cases to be recorded. but I am not aware that any instances of the employment of vascular grafting have been published in Great Britain.

CASE 1. Popliteal aneurysm treated by excision; restoration of arterial continuity by a vein graft. - The patient, a man aged 49, was admitted to the Royal Infirmary, Glasgow, on May 1st, 1912, complaining of a "throbbing swelling behind the left knee." He stated that he had had all the infective diseases of childhood, and an attack of rheumatism at the age of 24 years, when he was confined to bed for four weeks. He denied having had syphilis. He was married; his wife had had four children, all of whom were well, and one miscarriage between the second and third labours. He first observed a swelling behind the left knee eight months before his admission to hospital, and described it as being at that time "about the size of a marble" and "throbbing like a pulse." Since then the swelling had enlarged steadily if slowly. There was a history of his having fallen backwards over some scrap-iron, and of striking the back of the left knee, about four months prior to his discovery of the swelling ; but this injury produced no sign of any bruising of the tissues, and did not lay him off work. He consulted his own medical attendant, Dr. W. Campbell, of Springburn, regarding the tumour some three months before he came to hospital, and was then advised by him to seek admission, but did not do so, and continued at his work until April 30th, the day before he came into $m y$ ward.

The patient was of medium build; his lungs, liver, and spleen appeared to be normal. The heart was slightly enlarged, and there was a double murmur heard over the aortic area. The left popliteal space was occupied by an oval-shaped swelling which had an expansile pulsation. The skin over it was tense and glazed. A thrill was felt over the tumour, and by auscultation a harsh bruit could be heard over it. The circumference of the two knee-joints, measured at the centre of the patella, was-right knee, $33 \mathrm{~cm}$; left knee, $36.5 \mathrm{~cm}$. The pulse in the left posterior tibial artery felt at the ankle was sluggish and behind that of the right tibial in point of time. The superficial veins of both limbs up to the knee-joints were decidedly varicose, but those of the right leg were as bad as those of the left. The diagnosis of aneurysm was quite clear. The patient's blood gave a very positive Wassermann's reaction.

On May 16th the aneurysm was excised; it lay external to the popliteal vein and the nerve. The vein was closely adherent to the sac throughout its length and lay deeper than the sac-i.e., nearer the femoral than the superficial aspect of the sac. The sac itself was for the most part empty, but in its proximal portion contained some firm clot, and at this part was closely adherent to the surrounding structures. The artery was divided at each side immediately beyond the origin of the sac, the distance between the two lines of section being fully two inches; the vessel was clamped first on each side of the sac. I had hoped that it might be possible to attempt an end-to-end anastomosis of the two segments of the vessel; but even with the kneejoint flexed it was not possible to approximate the two ends nearer than to one inch of one another. Rather than ligate the two ends of the artery, I decide to attempt to maintain the continuity of the direct flow of arterial blood by grafting a portion of the saphena vein to fill the gap. Accordingly four inches of the internal saphena vein were removed from the middle of the right thigh and sutured to the eut ends of the artery by Carrel's method of eiroular suture; care was taken that the distal end of the segment of vein was sutured to the proximal segment of the artery in case there might be a valve in the portion of vein used, which would have seriously interfered with the passage of the blood through it. The length of vein excised exactly fitted the space between the two cut ends of the artery, which had retracted considerably.

The clamp that had been placed upon the distal side of the aneurysm was first removed, but the blood did not fill up, or even run into, the artery and grafted vein as I had expected, showing, I believe, that the collateral circulation was not efficient. After waiting several minutes to see if the backward flow of blood would fill up the vessels, but without result, the proximal clamp was removed from the artery, and at once blood rushed through the graft into the distal arteries. Not one drop of blood escaped at either of the lines of suture, so that nothing further was required than to suture up the wound in the overlying tissues and to apply a dressing. The limb was placed upon a posterior splint. From the moment, practically, of removing the proximal clamp from the artery the posterior tibial pulse in the two limbs seemed to be equal in force and time. The patient was kept in bed for three weeks and went home on June 14th, and at that time the pulsations along the course of the grafted vein were easily seen as well as felt.

The aneurysm measured $3 \frac{1}{1}$ in. Iongitudinally by $2 \frac{1}{2}$ in. transversely, and was sacculated. Looking into its interior the two openings of the artery are readily seen, separated by a distance of about two inches, but between these two openings no trace whatever of any furrow or trough suggesting the remainder of the lumen of the artery is to be made out.

The patient resumed his ordinary work on August 1st, 1912, and has continued steadily at it since. He has been seen on several occasions; the last time was Feb. 7th, when he was brought before the Glasgow Medico-Chirurgical Society. The posterior tibial pulses appear to be equal in time and force, and the pulsations of the popliteal artery can still be seen as well as felt. The grafted vein, therefore, remains perfectly patent.

CASE 2. Traumatic aneurysm of the brachial artery; veingrafting.-The patient, a lad aged 19, a blacksmith, was admitted to the Royal Infirmary on Feb. 24th, 1913, complaining of a swelling at the right elbow and inability to use the arm. 'The patient's medical history was good; he could not remember any previous illness. Five weeks before admission to hospital he had received an injury to the right upper limb; he was working at a steel railway rail when a fragment of steel flew off and penetrated the tissues just above his right elbow anteriorly. The small wound bled profusely at the time; a dressing was applied, and by the time he got home a swelling of some size had developed in front of the right elbow, causing him much pain at first. The swelling was at first soft, but as it became much firmer the pain practically went away. He thought that the swelling was not very much larger now than it was by the evening of the dar of the accident. The patient was sent to me by Dr. W. L. Cullen, of St. Boswells.

There was a swelling of some extent occupying the bend of the right elbow, hard, and brawny rather to feel, extending up into the arm and down into the forearm. In the skin about one inch above the line of the articulation, and just inside the mesial line of the limb, there was a small scar half an inch long, the result of the wound. The swelling was most prominent to the inner aspect of the limb and was fairly well defined. A faint thrill could be felt over the inner portion, and a bruit could be heard over the greater part of the tumour. Pulsation could not be detected. The right radial pulse was smaller than the left, and was decidedly delayed. There was no loss of sensation in the hand or forearm. The elbow-joint could not be fully extended, and could not be flexed actively more than to make an angle, open forwards, of about $120^{\circ}$, owing to the presence of the tumour, and any passive attempt to flex further was associated with pain. The hand was held about midway between pronation and supination, very little movement in either direction being permitted. There was no varicosity of the veins of the limb. An examination of the part under $X$ rays showed the presence of a small foreign body lying close against the humerus immediately above the coronoid fossa.

On the evening of Feb. 26th, although the patient had 
been kept in bed with the arm supported in a sling, there was a sudden access of really severe pain in the region of the affected elbow and forearm; it made him writhe about and produced profuse sweating, and was associated with a decided enlargement of the tumour. His temperature rose to $100^{\circ} \mathrm{F}$. As this increase of tension was either due to a fresh hæmorrhage or to infection I decided to operate on the following day. Incision was made over the most prominent part of the tumour. In trying to avoid a superficial vessel, median basilic (?) vein, another, lying immediately beneath the greatly stretched aponeurosis, was split for fully an inch, which turned out later to be the brachial artery. A fairly large space was opened into containing for the most part blood clot, but a certain amount of quite recently effused blood. There was no evidence of infection of the tissues. The clot was cleared out; some of it was lost, but what was collected weighed $3 \frac{1}{2} \mathrm{oz}$. It lay behind and to the inner aspect of the biceps and extended down beneath the pronator teres in the forearm; the small fragment of steel was found comparatively easily and was removed. The brachial artery matted together with its venæ comites formed a firm cord which had been lying upon the front of the clot and immediately under the deep aponeurosis and bicipital fascia. The median nerve was found lying at a plane much posterior to that of the artery. The artery had either to be tied or an attempt made with a graft to preserve the vascular continuity, and I decided for the latter. Owing to the matting of the vessels it was necessary to remove about $6 \mathrm{~cm}$. of their length in order to obtain a portion of the artery suitable for suturing. A piece of the right internal saphena vein, $8 \mathrm{~cm}$. long, was removed from the lower third of the thigh and sutured by Carrel's method to the two cut ends of the artery. The lumen of the artery was so small compared to that of the vein that the suturing was rather troublesome. When the circulation was allowed to go free again blood ran freely and easily through the grafted piece of vein, and a good pulse was felt in the radial artery at the wrist. The biceps was sutured as far over to the inner side of the arm as possible, to give support to the vessel, and the wound was closed. The arm was put up at a right angle.

The patient made an excellent recovery; he was allowed out of bed on March 17th. At this date the right radial pulse was very good and could be stopped at once by digital pressure over the intercondylar line in the upper arm, returning as soon as the pressure was relieved, although no pulsation could be felt at the front of the elbow owing to the brawny condition of the tissues which was still present. This was greatly lessened by massage, and the pulsations in the grafted ressel were quite readily recognised before the patient left hospital on March 24th. The movements at the elbow had greatly improved at this time also.

The patient was seen again on June 7th, when the state of the limb was as follows. He was able to flex the right elbowjoint completely and to extend it so that the forearm made with the upper arm an angle, open forwards. of $150^{\circ}$, the limitation of the extension being due to the thickening around the brachialis anticus muscle above the joint. Sensation over the fingers, hand, and forearm was normal. Pulsation in the grafted segment was readily felt as far down as the line of the articulation, practically as far as could be made out in the uninjured arm, while pressure over this part of the grafted vein arrested the radial pulse completely and at once. The patient started his work, as a blacksmith, again on May 12th, and felt quite able for it; he is making his former wage.

Glasgow.

Second Guildhali School Conference.Among those who are expected to take part in the Second Guildhall School Conference on Diet, Cookery, and Hygiene on June 30th and July 1st are Sir Arthur H. Downes, medical inspector (Poor-law), Local Government Board; Dr. R. A Lyster, county and school medical officer, Hampshire county council ; Dr. A. D. Edwards, medical officer of health and school medical officer, Bournemonth ; Dr. E. Bertram Smith, medical officer of health, North Essex united districts; and Dr. R. T. Herdman, deputy county medical officer and assistant school medical officer, Bedfordshire county council. Particulars of membership may be obtained from the Secretary, Schools Committee, 178, St. Stephen's House, Westminster.

\section{SENSITISED VACCINE IN ACUTE BAC-} TERIAL INFECTION.

\author{
RESULTS OBTAINED IN A SERIES OF CASES. ${ }^{2}$
}

Bx M. H. GORDON, M.A., M.D. Oxon., B.Sc.,

ASSISTANT PATHOLOGIST TO ST. BARTHOLOMEW'S HOSPITAL.

Sensitised Vaccine.

For the specific treatment of cases suffering from an acute bacterial infection two chief methods are at present in use. Either an immune serum containing antibodies specific for the infecting micro-organism is given, or an attempt is made to stimulate the patient's system to the active production of antibody by giving him a vaccine of the infecting micro-organism. From the practical standpoint each of these methods has its advantages, and koth have shortcomings. Apart from the possibility of giving rise to anaphylactic shock or serum sickness, immune sera, with the exception of diphtheria antitoxin and one or two others. have over and over again proved disappointing clinically. Ordinary vaccines, again, have the disadvantage that, before the period of increased immunity which they evoke is reached, the patient has to pass through a phase during which his resistance is actually lowered. While it is true that this negative phase may in many cases be minimised: and rendered invisible clinically by using a small initial dose, by increasing the dose slowly, and by properly interspacing the doses, the risk is nevertheless a real one; and those who have had much experience of vaccine treatmentespecially in acute cases-must occasionally have seen instances in which, owing to the specific hypersensitiveness of the patient, this diminution of resistance has proved a serious matter.

The law discovered by Ehrlich and Morgenroth, that every cell when brought in contact with its antibody fixes this antibody to the exclusion of all else, rendered it possible for Besredka to introduce a method of immunising that promised to form a step forward. This method is as follows. By the simple process of bringing the vaccine into contact with homologous immune serum the bacterial cells attract to themselves their specific antibodies present in the serum and become coated therewith. After sufficient time has elapsed for the adsorption of antibody to take place the bacterial cells are centrifuged out, the supernatant serum is removed and replaced by saline, the deposited bacteria are shaken up and again centrifuged out. Thus all traces of serum may be removed and a deposit is obtained which consists entirely of bacterial cells coated with their specific antibodies. This is the sensitised vaccine of Besredka.

There are one or two practical points that may be mentioned. It is advisable to count the vaccine before bringing it into contact with the immune serum. It is convenient to carry out all the stages in a graduated centrifuge tube. The vaccine is best killed after sensitisation, and this may be done by adding a suitable antiseptic to the saline with which the terminal washing is effected. Besredka appears to be in favour of not killing the vaccine at all, but of giving sensitised vaccine alive. Broughton-Alcock has given living sensitised typhoid bacilli, streptococci, and staphylococci to patients without reaction. This measure, however, implies great confidence in the sensitising properties of the sera used. Personally I have hitherto preferred to use sensitised vaccines in which the bacteria have been killed by phenol; but I have given living sensitised streptococci to two cases of ulcerative endocarditis without harmful effect.

The question now arises, What is the advantage of sensitising a vaccine? Surely a vaccine treated with immune serum in this way is neutralised and rendered inert? The answer to these questions is furnished by the results of the numerous experiments on animals that have been made by various independent observers since 1902, when Besredka: introduced the method. These experiments show that the first great effect of sensitisation is to reduce the toxicity of a. vaccine.

Examples. - The plague bacillus retains a good deal of its pathogenicity even when killed by heat. Thus $1 / 10$ to $1 / 15$ c.c. of a 48 hours agar culture of plague killed by heat 1 A paper read betore the Therapeutical Section of the Royal Societ5
of Medicine, May 20th, 1913. 